\title{
RIGIDITY AND CONVEXITY OF HYPERSURFACES IN SPHERES
}

\author{
M. P. DO CARMO \& F. W. WARNER
}

\section{Introduction}

We shall consider isometric immersions $x: M^{n} \rightarrow X^{n+1}$ of a compact, connected, orientable, $n$-dimensional $(n \geq 2), C^{\infty}$ Riemannian manifold $M^{n}$ in a simply connected Riemannian manifold $X^{n+1}$ of constant sectional curvature. When $X^{n+1}$ is the Euclidean space $E^{n+1}$, the following results, usually associated with the names of Hadamard and Cohn-Vossen, are known.

If $M^{n}$ has non-negative sectional curvatures, then

(a) $x\left(M^{n}\right) \subset E^{n+1}$ is the boundary of a convex body; in particular, $x$ is an embedding and $M^{n}$ is diffeomorphic to the unit sphere $S^{n} \subset E^{n+1}$.

(b) If $y: M^{n} \rightarrow E^{n}$ is another isometric immersion, then $x$ and $y$ differ by a rigid motion (isometry) of $E^{n+1}$.

Part (a) of the above result, for $n=2$ and positive curvature, was first proved by Hadamard [8]. The case $n=2$ with non-negative curvature was considered by Chern-Lashof [6], and the result for arbitrary $n$ follows from papers by van Heijenoort [16] and Sacksteder [14]; a simple direct proof can be found in do Carmo-Lima [7].

Part (b) for $n=2$ and positive curvature is the famous Cohn-Vossen theorem; a simple proof due to Herglotz may be found in [5]. The case $n=2$ with nonnegative curvature was considered by K. Voss [17] and, independently, by Pogorelov [11]. A proof for the general case follows from Sacksteder [15, Theorem V].

In this paper we shall primarily consider the case where $X^{n+1}$ is the unit sphere $S^{n+1} \subset E^{n+2}$ with its canonical metric of constant sectional curvature equal to one. (We indicate briefly in $\S 5$ the situation obtained when $X^{n+1}$ is a simply connected manifold of constant negative curvature.) A natural substitute for the above condition on the sectional curvatures is the requirement that the sectional curvatures of $M^{n}$ be greater than or equal to one (the curvature of the ambient space). We shall prove that the Hadamard and Cohn-Vossen results have exact analogues in this case. Precisely, we prove the following

1.1. Theorem. Let $x: M^{n} \rightarrow S^{n+1}$ be an isometric immersion of a compact, connected, orientable $n$-dimensional $C^{\infty}$ Riemannian manifold into the

Communicated by S. S. Chern, April 17, 1969. The first author was a Guggenheim fellow supported partially by NSF grant GP 6974 and C.N.Pq., and the second author was supported in part by NSF grants GP 6895 and GP 6974. 
$(n+1)$-sphere of constant sectional curvature equal to one, and assume that all sectional curvatures of $M^{n}$ are greater than or equal to one.

(a) Then $x$ is an imbedding, $M^{n}$ is diffeomorphic with $S^{n}$, and $x\left(M^{n}\right)$ is either totally geodesic or contained in an open hemisphere; in the latter case $x\left(M^{n}\right)$ is the boundary of a convex body in $S^{n+1}$.

(b) If $y: M^{n} \rightarrow S^{n+1}$ is another isometric immersion, then there is an isometry $\alpha$ of $S^{n+1}$ such that $\alpha \circ x=y$.

\subsection{Remarks.}

(a) If the sectional curvatures of $M^{n}$ are strictly greater than one, then the case 1.1(b) for $n>2$ follows trivially from the classical rigidity theorem [4, p. 211].

(b) Assuming the truth of 1.1(a), the assertion 1.1(b) for $n=2$ follows from Pogorelov [13, p. 105].

(c) In [12] Pogorelov proves a very general rigidity theorem which includes our assertion 1.1(b) for $n=2$ and curvature strictly greater than one.

\section{The Beltrami maps}

The proof of Theorem 1.1 will require extensive use of the Beltrami maps in transforming problems on the sphere $S^{n+1}$ to problems in a Euclidean space, so we shall devote this section to defining these maps and deriving their relevant properties.

Let $\nu \in S^{n+1}$, and let $H_{\nu}$ denote the open hemisphere of $S^{n+1}$ centered at $\nu$. The Beltrami map $\beta_{\nu}$ is the diffeomorphism of $H_{\nu}$ onto the hyperplane $S_{\nu} \subset E^{n+2}$ tangent to $S^{n+1}$ at $\nu$ obtained by central projection. In the special case in which $\nu$ is the north pole $(0, \cdots, 0,1)$, we shall denote the corresponding Beltrami map by $\beta_{0}$, the open northern hemisphere by $H_{0}$, and the tangent hyperplane at the north pole by $S_{0}$. If points of $H_{0}$ are denoted by $(n+2)$-tuples $\left(\xi_{1}, \cdots\right.$, $\left.\xi_{n+2}\right)$ of real numbers with $\sum \xi_{i}^{2}=1$ and $\xi_{n+2}>0$, then the Beltrami map $\beta_{0}$ is explicitly given by

$$
\beta_{0}(\xi)=\left(\xi_{1} / \xi_{n+2}, \xi_{2} / \xi_{n+2}, \cdots, \xi_{n+1} / \xi_{n+2}, 1\right) .
$$

It is well known (and easily checked) that these maps are geodesic, that is, they map geodesics of the sphere setwise onto geodesics (straight lines) in the tangent hyperplanes (where we consider each tangent hyperplane of $S^{n+1}$ to be equipped with the canonical Riemannian structure inherited from $E^{n+2}$ ). We shall use $\beta_{\nu}$ to transform hypersurfaces of $H_{\nu}$ with sectional curvatures greater than or equal to one into hypersurfaces of $S_{\nu}$ with sectional curvatures greater than or equal to zero, and vice versa. To see that $\beta_{\nu}$ indeed does have this effect we first prove two lemmas (Lemmas 2.1 and 2.2 below) for which we need a few definitions.

By the height function for an oriented hypersurface at a point $p$ we shall mean the function defined on a neighborhood of the origin in the tangent plane 
of the hypersurface at $p$ and assigning to each point of this neighborhood the height, with respect to the oriented normal, of the hypersurface above its tangent plane as measured in a normal coordinate system about $p$ in the ambient manifold. Since the origin is a critical point for such a height function, the matrix of second partial derivatives at the origin determines a well defined bilinear form in the tangent space of the hypersurface at $p$ called the hessian of the height function, and as well known [4, p. 198], this hessian form is the negative of the second fundamental form for the hypersurface at that point. By the eigenvalues of the second fundamental form, or the hessian form, we shall mean the eigenvalues of the symmetric linear transformations in the tangent space induced by these forms relative to the given inner product.

2.1. Lemma. Let $m$ be a point on an oriented hypersurface in $E^{n+1}$, and suppose that in a neighborhood of $m$ on the hypersurface the eigenvalues of the second fundamental forms do not have different signs. Then there is a neighborhood of $m$ on the hypersurface which lies on one side of the tangent hyperplane at $m$.

Proof. For simplicity we may assume that $m$ is the origin 0 of $E^{n+1}$ and that the hypersurface is represented near 0 by the equation $z=f(x)$, where $f$ is a real-valued function defined on the open unit disc $D$ about the origin in $E^{n} \subset E^{n+1}$ with $f(0)=0$ and all first partial derivatives of $f$ vanishing at 0 . We may also assume that the hypersurface is oriented so that the oriented unit normal at 0 is the unit vector in the positive " $z$ direction", and without loss of generality we may assume that all the eigenvalues of the second fundamental forms on this piece of hypersurface are negative or zero. Thus, at any point $q$ on the hypersurface, the eigenvalues of the hessian of the height function are all positive or zero. We shall prove the hypersurface lies on one side of its tangent plane at 0 ; indeed we shall prove $f(x) \geq 0$ for all $x \in D$, and to this end we argue by contradiction. Suppose there is a point $p \in D$ where $f(p)<0$. Then there must exist a $t_{0}$ with $0 \leq t_{0} \leq 1$ such that the function $h(t)=f(t p)$ has a negative second derivative at $t_{0}$; thus $h^{\prime \prime}\left(t_{0}\right)<0$. Consider now the corresponding curve $P(t)=(t p, f(t p))$ on the hypersurface, and let $h_{0}$ denote the height function of the hypersurface above its tangent hyperplane $T_{0}$ at $P\left(t_{0}\right)$, and let $X$ be the parallel vector field on $T_{0}$ determined by $P^{\prime}\left(t_{0}\right)$. We shall show that the second derivative $X^{2}\left(h_{0}\right)$ at the origin of $T_{0}$ is negative and this will contradict the fact that the hessian of $h_{0}$ at the origin of $T_{0}$ is positive semidefinite. Now the height of $P(t)$ above the tangent plane $T_{0}$ is given by

$$
h_{0}(t)=\left[P(t)-P\left(t_{0}\right)\right] \cdot n,
$$

where $n$ is the oriented unit normal at $P\left(t_{0}\right)$, and the second derivative $X^{2}\left(h_{0}\right)$ at the origin in $T_{0}$ is easily seen to be $h_{0}^{\prime \prime}\left(t_{0}\right)$. Now $h_{0}^{\prime \prime}\left(t_{0}\right)=P^{\prime \prime}\left(t_{0}\right) \cdot n$, which is $\left(0, h^{\prime \prime}\left(t_{0}\right)\right) \cdot\left(-\operatorname{grad} f\left(t_{0} p\right),+1\right)$, which equals $h^{\prime \prime}\left(t_{0}\right)$. Since this, as we have seen, is negative, the proof is complete. 
We also need the version of the above lemma for hypersurfaces of a sphere.

2.2. Lemma. Let $m$ be a point on an oriented hypersurface in $S^{n+1}$, and suppose that in a neighborhood of $m$ on the hypersurface the eigenvalues of the second fundamental forms do not have different signs. Then there is a neighborhood of $m$ on the hypersurface which lies on one side of the tangent hypersphere at $m$.

Proof. We argue by contradiction. Suppose the hypersurface cuts its tangent hypersphere at $m$, and restrict attention to a neighborhood $U$ of $m$ on the hypersurface on which the second fundamental forms are everywhere, say, negative semi-definite (which can be achieved by a change of orientation if necessary), so that the hessians of the height functions are all positive semidefinite. Transfer this hypersurface, together with its orientation, to the Euclidean space $S_{m}$ via the Beltrami map $\beta_{m}$.

Since the hypersurface $\beta_{m}(U)$ cuts its tangent plane at $m$, according to the preceding lemma there must be a point $\beta_{m}(p) \in \beta_{m}(U)$ at which the hessian of the height function has a negative eigenvalue, and therefore in the direction of the corresponding eigenvector the hypersurface $\beta_{m}(U)$ locally lies on the side of its tangent plane at $\beta_{m}(p)$ opposite from the oriented normal direction. We claim the hessian of the height function for $U$ at $p$ also has a negative eigenvalue, which will provide us with a contradiction. Since the hessian of the height function for $\beta_{m}(U)$ at $\beta_{m}(p)$ has a negative eigenvalue, the surface $\beta_{m}(U)$ and its tangent plane at $\beta_{m}(p)$ have contact of order exactly 1 in the corresponding eigen-direction. That is, there is a pair of curves, one on the surface $\beta_{m}(U)$ and one on its tangent plane at $\beta_{m}(p)$ both passing through $\beta_{m}(p)$ tangent to this eigendirection and having contact of order exactly one at $\beta_{m}(p)$ and no such pair will have higher order contact. Now contact is preserved under diffeomorphism [9, p. 80], so the hypersurface $U$ and its tangent hypersphere at $p$ have contact of order exactly 1 in the corresponding direction. Therefore in this direction the height function at $p$ must have a non-zero second derivative, which is necessarily negative since in this direction the hypersurface $U$ lies for a while on the side of its tangent hypersphere at $p$ opposite from the oriented normal direction. Thus the hessian of the height function at $p$ is not positive semi-definite, which is a contradiction.

We are now in a position to describe the effect which the Beltrami maps have on sectional curvatures. If $X$ is a Riemannian manifold, $K_{X}$ shall denote the function which assigns to each 2-plane tangent to $X$ its sectional curvature.

2.3. Proposition. Let $\nu \in S^{n+1}$, let $X \subset H_{\nu}$ be a hypersurface, and let $\dot{X}$ denote the hypersurface $\beta_{\nu}(X)$ in $S_{\nu}$. Then $K_{X} \geq 1$ everywhere if and only if $K_{\widetilde{X}} \geq 0$ everywhere. Moreover, if $K_{X} \geq 1$, and if the rank of the second fundamental form for $X$ at $p \in X$ is $r, 0 \leq r \leq n$, then the rank of the second fundamental form for $\bar{X}$ at $\beta_{\nu}(p)$ is also $r$.

Proof. Let $p \in X$ and assume $K_{X} \geq 1$. Recall that the sectional curvature $K_{X}(P)$ of a 2-plane $P \subset X_{p}$, where $X_{p}$ denotes the tangent space of $X$ at $p$, is 
given by

$$
K_{X}(P)=1+S_{X}(p)(x, x) S_{X}(p)(y, y)-\left(S_{X}(p)(x, y)\right)^{2},
$$

where $S_{X}(p)$ is the second fundamental form for the hypersurface $X$ at $p$, and $\{x, y\}$ is an orthonormal basis of $P$. The sign of $S_{X}(p)$ depends on a choice of one of the two unit normals at $p$-we assume a continuous choice of unit normals on a neighborhood of $p$. Suppose first that not all of the eigenvalues of $\mathrm{S}_{X}(p)$ are zero. Since $K_{X} \geq 1$, it follows that all the non-zero eigenvalues of $S_{X}(p)$ will have the same sign, and nearby $p$ all the non-zero eigenvalues of the second fundamental forms for $X$ will have that same fixed sign. Therefore from Lemma 2.2, the hypersurface $X$ locally lies on one side of the tangent hypersphere through $p$. Since the Beltrami map $\beta_{\nu}$ sends this hypersphere to a hyperplane in $S_{\nu}, \bar{X}$ must locally lie on one side of its tangent hyperplane at $\beta_{\nu}(p)$. Thus all sectional curvatures at $\beta_{\nu}(p) \in X$ are $\geq 0$, for if there were a negative sectional curvature at $\beta_{\nu}(p), \dot{X}$ would lie on both sides of its tangent hyperplane at $\beta_{\nu}(p)$. Now suppose that all eigenvalues of $S_{X}(p)$ are zero. If they vanish identically on an entire neighborhood of $p$, then $X$ is totally geodesic near $p$, so $\bar{X}$ is totally geodesic and in particular flat near $\beta_{\nu}(p)$. If each neighborhood of $p$ contains points at which there are non-zero eigenvalues of the second fundamental form for $X$, then there is a sequence $\left\{p_{i}\right\}$ in $X$ converging to $p$, for which we already know that the sectional curvatures at $\beta_{\nu}\left(p_{i}\right)$ are all $\geq 0$. Hence by continuity, the sectional curvatures at $\beta_{\nu}(p)$ are all $\geq 0$.

A similar argument in the reverse direction shows the converse, namely, if $K_{\widetilde{X}} \geq 0$, then $K_{X} \geq 1$.

Now assume that the rank of $S_{X}(p)$ is $r$. Equivalently the hessian of the height function at $p$ has rank $r$. If in addition $K_{X} \geq 1$, then there is an $r$-dimensional subspace $A$ of $X_{p}$, on which the hessian is either positive or negative definite. It follows that in each direction in $A$ the hypersurface $X$ has contact of order exactly 1 with the tangent hypersphere through $p$. Since contact is preserved by the diffeomorphism $\beta_{\nu}$, there is an $r$-dimensional subspace of $\bar{X}_{\beta_{\nu}(p)}$ along which $\bar{X}$ has contact of order exactly 1 with the tangent hyperplane through $\beta_{\nu}(p)$. Consequently, the rank of the hessian of the height function for $\bar{X}$ at $\beta_{\nu}(p)$ must be at least $r$, so that $\operatorname{rank} S_{\widetilde{X}}\left(\beta_{\nu}(p)\right) \geq \operatorname{rank} S_{X}(p)$. Reversing the argument we obtain the opposite inequality. Hence $\operatorname{rank} S_{\widetilde{X}}\left(\beta_{\nu}(p)\right)=\operatorname{rank} S_{X}(p)$.

\subsection{Remarks.}

(a) It follows from Proposition 2.3 that a point in $X$ at which all sectional curvatures are strictly greater than one, is mapped under $\beta_{\nu}$ into a point of $\bar{X}$ at which all sectional curvatures are strictly greater than zero, and vice versa.

(b) It would have been easier in Proposition 2.3 if we could have concluded from the curvature assumption on $X$ that $X$ was locally convex in the sphere; for then since the Beltrami map $\beta_{\nu}$ is geodesic, it would follow directly that $\bar{X}$ would be locally convex in the Euclidean space $S_{\nu}$, implying that all sectional 
curvatures for $\hat{X}$ are non-negative. However, it should be observed that without some global condition, the fact that $K_{X} \geq 1$ does not suffice to ensure the local convexity of $X$ in $S^{n+1}$. The following is adapted from an example in [14]. Let $z=x^{3}\left(1+y^{2}\right)$ be a surface $\Sigma$ in $E^{3}$ defined in a small neighborhood of the origin, let $\alpha: E^{3} \rightarrow S_{\nu}$ be an isometry preserving the origins, and consider the surface $\beta_{\nu}^{-1}(\alpha(\Sigma))$ in $S^{3}$. It is easily seen that the curvature of $\Sigma$ is greater than or equal to zero, and hence, by Proposition 2.1 the curvature of $\beta_{\nu}^{-1}(\alpha(\Sigma))$ is greater than or equal to one. On the other hand, $\Sigma$ "crosses" its tangent plane at the origin; hence it is not locally convex at 0 . Since the map $\beta_{\nu}$ is geodesic, it follows that $\beta_{\nu}^{-1}(\alpha(\Sigma))$ is not locally convex at $\beta_{\nu}^{-1}(0)$. Our Theorem 1.1 implies that such a piece of surface cannot be extended to a compact surface in $S^{3}$ with curvature greater than or equal to one.

2.5. Lemma. Let $x: M \rightarrow S^{n+1}$ be an isometric immersion of a complete Riemannian manifold $M$ into the sphere $S^{n+1}$, and let $\nu \in S^{n+1}, N=x^{-1}\left(H_{\nu}\right)$, and $\tilde{x}=\beta_{\nu} \circ x \mid N$. Then the Riemannian structure on $N$ induced by the immersion $\tilde{x}: N \rightarrow S_{\nu}$ is also complete.

Proof. Let $\left\{p_{i}\right\}$ be a Cauchy sequence in $N$ with respect to the metric induced by $\tilde{x}$, and let $q_{i}=x\left(p_{i}\right)$. Since $\bar{H}_{\nu}$ is compact, the $q_{i}$ 's, or a subsequence, converge to a point $q \in \bar{H}_{\nu}$. Since $x$ is an isometric immersion of the complete Riemannian manifold $M$ into $S^{n+1}, q=x(p)$ for some $p \in M$. Now $q$ actually belongs to $H_{\nu}$, for if $q$ were in the equator $\bar{H}-H$, then the sequence $\left\{\beta_{\nu}\left(q_{i}\right)\right\}$ would diverge to $\infty$ in $S_{\nu}$, which is impossible in view of the assumption that $\left\{p_{i}\right\}$ is a Cauchy sequence in $N$ with respect to the metric induced by $\tilde{x}=\beta_{\nu} \circ x$. Thus $q \in H_{\nu}$, so $p \in N$. Since the sequence $\left\{\beta_{\nu}\left(q_{i}\right)\right\}$ converges to $\beta_{\nu}(q)$, it follows that the Cauchy sequence $\left\{p_{i}\right\}$ converges to $p$ in the metric on $N$ induced from $\tilde{x}$.

This completes the information we shall need concerning the Beltrami maps, and we now begin the proof of Theorem 1.1.

\section{Proof of Theorem 1.1 (a)}

If all sectional curvatures on $M^{n}$ are equal to one, then according to O'Neill [10] $x$ is an isometric imbedding of $M^{n}$ onto a great $n$-sphere in $S^{n+1}$, from which the theorem follows for this special case. If not all sectional curvatures are one, we shall first prove that there is at least one point of $M^{n}$, at which all sectional curvatures are greater than one. Suppose this is not the case (that is, we are assuming that there are sectional curvatures greater than one, but at no point are all sectional curvatures greater than one); we shall derive a contradiction. Let $p$ be a point of $M^{n}$, at which the second fundamental form for the immersion $x$ has maximal rank, say $r$. It follows from our assumptions that $1<r<n$. Let $\nu=x(p)$, and as before let $H_{\nu}$ be the open hemisphere centered at $\nu$, and $\beta_{\nu}$ the corresponding Beltrami map. Let $N_{\nu}=$ $x^{-1}\left(H_{\nu}\right), x_{\nu}=x \mid N_{\nu}$, and $\tilde{x}_{\nu}=\beta_{\nu} \circ x_{\nu}$. Now $N_{\nu}$ has two Riemannian structures 
-one inherited from $M^{n}$, for which $x_{\nu}: N_{\nu} \rightarrow H_{\nu}$ is an isometric immersion, and the other with respect to which $\tilde{x}_{\nu}: N_{\nu} \rightarrow S_{\nu}$ is an isometric immersion. To distinguish these Riemannian structures we shall denote $N_{\nu}$ with the metric induced from $\tilde{x}_{\nu}$ by $\tilde{N}_{\nu}$. Then it follows from Proposition 2.3 and Lemma 2.5 that $\tilde{N}_{\nu}$ is a complete Riemannian manifold with sectional curvatures greater than or equal to zero and that the second fundamental form for $\tilde{N}_{\nu}$ has maximal rank $r$. According to Sacksteder [14], through each point of $\tilde{x}_{\nu}\left(\tilde{N}_{\nu}\right)$ there passes an $(n-r)$-dimensional plane $\Pi$ entirely contained in $\tilde{x}_{\nu}\left(\tilde{N}_{\nu}\right)$, along which the tangent hyperplane of $\tilde{x}_{\nu}\left(\tilde{N}_{\nu}\right)$ is constant and contains $\Pi$, moreover distinct $(n-r)$-planes in this collection are parallel. Under $\beta_{\nu}^{-1}$ these planes are mapped to $(n-r)$-dimensional great hemispheres contained in $x_{\nu}\left(N_{\nu}\right)$. Now it is a classical local fact that on a neighborhood $U$ of $x(p)$, where the rank of the second fundamental form is constantly equal to $r$, the distribution determined by the 0 -eigenspaces of the second fundamental form is integrable. The integral manifolds of this distribution must agree on $U$ with the above $(n-r)$-spheres; otherwise, the maximal rank of the second fundamental form for $N_{\nu}$ would be $<r$. It follows from another application of Sacksteder's theorem that for any $\nu^{\prime} \in U$ these $(n-r)$-spheres (more precisely, their intersections with $U$ ) must map under the Beltrami map $\beta_{\nu^{\prime}}$ to parallel planes in $S_{\nu^{\prime}}$. We thus have a contradiction; for given a collection of $(n-r)$-spheres in $S^{n+1}$, which are transformed under one Beltrami map into parallel planes, one can always find a nearby Beltrami map for which the images are no longer parallel. Thus, if there are sectional curvatures of $M^{n}$ greater than one, then there is at least one point of $M^{n}$ at which all sectional curvatures are greater than one.

Now let $p \in M^{n}$ be a point at which all sectional curvatures are greater than one. Then one can choose $\nu \in S^{n+1}$ such that $x(p)$ lies in the equater $\bar{H}_{\nu}-H_{\nu}$, and such that there is a neighborhood $V$ of $p$ in $M$ satisfying the condition $x(\bar{V}-\{p\}) \subset H_{\nu}$. We next prove that $x\left(M^{n}-\{p\}\right) \subset H_{\nu}$. Let $N_{\nu}$ be the connected component of $x^{-1}\left(H_{\nu}\right)$ containing $V-\{p\}$. As before, let $x_{\nu}=x \mid N_{\nu}$, and $\tilde{x}_{\nu}=\beta_{\nu} \circ x_{\nu}$, and let $\tilde{N}_{\nu}$ denote $N_{\nu}$ with the Riemannian structure induced by $\tilde{x}_{\nu}$. Then by Proposition 2.3 and Lemma $2.5, \tilde{N}_{\nu}$ is a complete Riemannian manifold with sectional curvatures greater than or equal to zero, and at some point $q \neq p$ in $V$, all sectional curvatures of $\tilde{N}_{\nu}$ are positive. Thus from the main theorem of [14] it follows that $\tilde{x}_{\nu}\left(\tilde{N}_{\nu}\right) \subset S_{\nu}$ is the boundary of a convex body which contains no complete line, and since $\tilde{N}_{\nu}$ is non-compact, $\tilde{N}_{\nu}$ is diffeomorphic to a Euclidean space. If we choose the neighborhood $V$ of $p$ to be homeomorphic to a disc, it follows from the above that the image $\tilde{x}_{\nu}(\partial V)$ of the boundary $\partial V$ of $V$ separates $\tilde{x}_{\nu}\left(\tilde{N}_{\nu}\right)$ into two connected components, one of which, say $W$, is bounded in $\mathrm{S}_{\nu}$. Let $m \in N_{\nu}-V$. Then we claim $\tilde{x}_{\nu}(m) \in W$. Assume the contrary, and choose a curve in $N_{\nu} \cup\{p\}$ joining $m$ to $p$, which "crosses" $\partial V$ only once. The image of this curve under $\tilde{x}_{\nu}$ starts in the unbounded component, crosses $\tilde{x}_{\nu}(\partial V)$ only once and becomes unbounded. This con- 
tradiction proves our claim. Thus no point of $\bar{H}_{\nu}-H_{\nu}$ is a limit point of $x_{\nu}\left(N_{\nu}-V\right)$, and hence no point of $\bar{H}_{\nu}-H_{\nu}$, except $x(p)$ it self, is a limit point of $x_{\nu}\left(N_{\nu}\right)$. Since $M^{n}$ is connected, it follows that $x\left(M^{n}-\{p\}\right) \subset H_{\nu}$ as we asserted.

Now we can choose $\nu^{\prime}$ near the $\nu$ used above so that $x\left(M^{n}\right)$ lies entirely in the open hemisphere $H_{\nu^{\prime}}$. Then $\beta_{\nu^{\prime}} \circ x: M^{n} \rightarrow S_{\nu^{\prime}}$ is an isometric immersion of a compact manifold with non-negative sectional curvatures in the induced metric. According to Sacksteder [14] (see also [7]), $\beta_{\nu}, x\left(M^{n}\right)$ is the boundary of a convex body in $S_{\nu^{\prime}}$. Applying $\beta_{\nu^{\prime}}^{-1}$ we immediately obtain the conclusions of part (a) of Theorem 1.1.

\section{Proof of Theorem $1.1(b)$}

We now assume that $M^{n}$ is a compact, connected, orientable, $C^{\infty}$ Riemannian manifold with $K_{M} \geq 1$, and that $x$ and $y$ are two isometric immersions of $M^{n}$ into $S^{n+1}$.

If all sectional curvatures of $M^{n}$ are equal to one, both $x$ and $y$ are isometric imbeddings of $M^{n}$ onto great $n$-spheres in $S^{n+1}$, from which it follows that there is an isometry $\alpha$ of $S^{n+1}$ such that $\alpha \circ x=y$.

If there are sectional curvatures of $M^{n}$ greater than one, then, as we observed in $\S 3$, both $x$ and $y$ are imbeddings, and both $x\left(M^{n}\right)$ and $y\left(M^{n}\right)$ lie in open hemispheres and are boundaries of convex bodies. Without loss of generality we can assume that both $x\left(M^{n}\right)$ and $y\left(M^{n}\right)$ lie in the open northern hemisphere $H_{0}$, and that both are "visible from the inside" at the north pole $e_{0}=(0, \cdots$, 0,1 ) (that is, any geodesic from the north pole meets each surface in exactly one point).

To prove rigidity we shall use a generalization to $n$-dimensions of a technique due to Pogorelov, which involves mapping the surfaces $x\left(M^{n}\right)$ and $y\left(M^{n}\right)$ to $E^{n+1}$ via special mixed Beltrami-like maps, applying known rigidity theorems in $E^{n+1}$, and then mapping back to the sphere.

Define $\tilde{x}: M^{n} \rightarrow E^{n+1}$ (similarly $\tilde{y}: M^{n} \rightarrow E^{n+1}$ ) by

$$
\tilde{x}(p)=\left[x(p)-e_{0}\left(x(p), e_{0}\right)\right] /\left(e_{0}, x(p)+y(p)\right) .
$$

Here we are using vector notation in $E^{n+2}$, and $($, ) denotes the standard Euclidean inner-product. Now $x, y, \tilde{x}$ and $\tilde{y}$ can each be considered as a vector-valued function on $M^{n}$ with values in $E^{n+2}$, so their differentials $d x, d y$, $d \tilde{x}$ and $d \tilde{y}$ can be considered as $E^{n+2}$-valued one-forms on $M$. Both $\tilde{x}$ and $\tilde{y}$ are $C^{\infty}$ imbeddings of $M^{n}$ into $E^{n+1} \subset E^{n+2}$, and further induce the same metric on $M^{n}$. For, in view of the facts that $(d x, d x)=(d y, d y)$ and $(d x, x)=(d y, y)$ $=0$, by an elementary calculation one can show that $(d \tilde{x}, d \tilde{x})=(d \tilde{y}, d \tilde{y})$. Denote $M^{n}$ with the metric induced from $\tilde{x}$ and $\tilde{y}$ by $\bar{M}^{n}$. Thus $\tilde{x}$ and $\tilde{y}$ are isometric imbeddings of $\bar{M}^{n}$ into $E^{n+1}$. It follows from Pogorelov's Theorem 3 [13, p. 63] that both $\tilde{x}\left(\tilde{M}^{n}\right)$ and $\tilde{y}\left(\tilde{M}^{n}\right)$ are locally convex hypersurfaces in 
$E^{n+1}$. Pogorelov's theorem is written for the special case $n=2$ but goes over nearly word for word for general $n$. Thus the sectional curvatures of $\bar{M}^{n}$ are all greater than or equal to zero, and since $\tilde{x}\left(\bar{M}^{n}\right)$ is a compact hypersurface of $E^{n+1}$, there is at least one point of $\bar{M}^{n}$ at which all sectional curvatures are strictly positive. By [14] or [7], both $\tilde{x}\left(\bar{M}^{n}\right)$ and $\tilde{y}\left(\bar{M}^{n}\right)$ bound convex bodies in $E^{n+1}$. Thus it follows from Theorem $\mathrm{V}$ of [15] that there exists an isometry $\tilde{\alpha}$ of $E^{n+1}$ such that $\tilde{\alpha} \circ \tilde{x}=\tilde{y}$.

We define maps $\rho_{1}$ and $\rho_{2}$ from $E^{n+1}$ into $S^{n+1}$ by

$$
\begin{aligned}
& \rho_{1}(p)=\frac{2 p+e_{0}\left(1+(\tilde{\alpha}(p))^{2}-p^{2}\right)}{\| \text { numerator } \|}, \\
& \rho_{2}(p)=\frac{2 p+e_{0}\left(1+\left(\tilde{\alpha}^{-1}(p)\right)^{2}-p^{2}\right)}{\| \text { numerator } \|},
\end{aligned}
$$

Here $p^{2}$ is used to denote the inner product $(p, p)$ in $E^{n+2}$. Now $\rho_{1}$ and $\rho_{2}$ are $C^{\infty}$, and a straightforward calculation shows that

$$
\begin{aligned}
& \rho_{1}(\tilde{x}(p))=x(p), \\
& \rho_{2}(\tilde{y}(p))=y(p)
\end{aligned}
$$

for all $p \in M^{n}$. The following argument due to Pogorelov shows that $\rho_{1}$, similarly $\rho_{2}$, is a $1: 1$ map. Suppose $p \neq q \in E^{n+1}$ but $\rho_{1}(p)=\rho_{1}(q)$. It follows immediately from the nature of the map $\rho_{1}$ that $p$ and $q$ must be parallel, so there exists a unit vector $v \in E^{n+1}$ such that $p=\lambda v$ and $q=\mu v$. Furthermore, the equality of $\rho_{1}(p)$ and $\rho_{1}(q)$ yields immediately the equality

$$
\left[1+(\widetilde{\alpha}(p))^{2}-\lambda^{2}\right] / \lambda=\left[1+(\widetilde{\alpha}(q))^{2}-\mu^{2}\right] / \mu .
$$

Consider $\tilde{\alpha}$ as a rotation $\tilde{\alpha}^{*}$ followed by a translation by the vector $c$. Then

$$
\begin{aligned}
& (\tilde{\alpha}(p))^{2}=\left(\tilde{\alpha}^{*}(\lambda v)+c\right)^{2}=\lambda^{2}+2\left(\tilde{\alpha}^{*}(\lambda v), c\right)+c 2, \\
& (\tilde{\alpha}(q))^{2}=\mu 2+2\left(\widetilde{\alpha}^{*}(\mu v), c\right)+c^{2},
\end{aligned}
$$

and using these, (3) becomes

$$
\frac{1+c^{2}}{\lambda}+2\left(\tilde{\alpha}^{*}(v), c\right)=\frac{1+c^{2}}{\mu}+2\left(\tilde{\alpha}^{*}(v), c\right) .
$$

Thus $\lambda=\mu$ and $p=q$, which is a contradiction. So $\rho_{1}$ is $1: 1$, and similarly $\rho_{2}$ is $1: 1$. It follows from the invariance of domain theorem that $\rho_{1}$ and $\rho_{2}$ are both open maps. Hence $\rho_{2} \circ \tilde{\alpha} \circ \rho_{1}^{-1}$ defines a 1:1 map $\alpha$ on some connected open neighborhood of $x\left(M^{n}\right)$ in $S^{n+1}$ and $\alpha(x(p))=y(p)$. We claim $\alpha$ extends to an isometry of $S^{n+1}$ (which will complete the proof of Theorem 1.1 (b)) and for this it is sufficient to prove $\alpha$ preserves distances. To this end, by following an 
argument of Pogorelov we show that

$$
\left(\rho_{1}(p)-\rho_{1}(q)\right)^{2}=\left(\rho_{2}(\widetilde{\alpha}(p))-\rho_{2}(\widetilde{\alpha}(q))\right)^{2} .
$$

Since the Euclidean distance between a pair of points on the sphere uniqully determines their spherical distance, this will complete the proof. To prove (5) it suffices to prove

$$
\left.\left(\rho_{1}(p)\right), \rho_{1}(q)\right)=\left(\rho_{2}(\tilde{\alpha}(p)), \rho_{2}(\widetilde{\alpha}(q))\right) .
$$

Let

$$
\begin{aligned}
& a(p)=2 p+e_{0}\left(1+(\tilde{\alpha}(p))^{2}-p^{2}\right), \\
& b(p)=2 \widetilde{\alpha}(p)+e_{0}\left(1+p^{2}-(\widetilde{\alpha}(p))^{2}\right) .
\end{aligned}
$$

Then

$$
\begin{aligned}
\rho_{1}(p) & =a(p) / \sqrt{(a(p))^{2}}, \\
\rho_{2}(\tilde{\alpha}(p)) & =b(p) \sqrt{(b(p))^{2}} .
\end{aligned}
$$

So to prove (6) it suffices to prove that for an arbitrary pair of points $p, q \in E^{n+1}$,

$$
(a(p), a(q))=(b(p), b(q)) .
$$

Now

$$
\begin{aligned}
& (a(p), a(q))=4(p, q)+\left(1+\tilde{\alpha}(p)^{2}-p^{2}\right)\left(1+\tilde{\alpha}(q)^{2}-q^{2}\right), \\
& (b(p), b(q))=4(\tilde{\alpha}(p), \tilde{\alpha}(q))+\left(1+p^{2}-\tilde{\alpha}(p)^{2}\right)\left(1+q^{2}-\tilde{\alpha}(q)^{2}\right) .
\end{aligned}
$$

Write $\tilde{\alpha}$ as a rotation $\tilde{\alpha}^{*}$ and a translation by the vector $c$. Then

$$
\begin{aligned}
& \tilde{\alpha}(p)^{2}=p^{2}+2\left(c, \tilde{\alpha}^{*}(p)\right)+c^{2}, \\
& \tilde{\alpha}(q)^{2}=q^{2}+2\left(c, \tilde{\alpha}^{*}(q)\right)+c^{2},
\end{aligned}
$$

and

$$
(\tilde{\alpha}(p), \tilde{\alpha}(q))=(p, q)+\left(c, \tilde{\alpha}^{*}(p)+\tilde{\alpha}^{*}(q)\right)+c^{2} .
$$

Substituting these expression in (8), one immediately obtains (7). This completes the proof of Theorem 1.1.

\section{Ambient manifold of constant negative curvature}

Let $X^{n+1}$ now be a simply connected manifold with constant sectional curvature equal to -1 (the hyperbolic space), and let $x: M^{n} \rightarrow X^{n+1}$ be an isometric immersion of a compact, connected orientable $C^{\infty}$ Riemannian 
manifold $M^{n}$ whose sectional curvatures are all greater than or equal to -1 . $X^{n+1}$ possesses a global Beltrami coordinate system giving a geodesic mapping of $X^{n+1}$ onto a half space in $E^{n+1}$ [3, p. 435]. If we transfer the hypersurface $x\left(M^{n}\right)$ to $E^{n+1}$ via this Beltrami map, then by arguments similar to those in Lemma 2.2 and Proposition 2.3 we obtain a compact hypersurface of $E^{n+1}$ with sectional curvatures $\geq 0$. Applying the known results for $E^{n+1}$ and transferring back to $X^{n+1}$ we conclude that $x$ is an imbedding, $M^{n}$ is diffeomorphic to $S^{n}$, and $x\left(M^{n}\right)$ bounds a convex body in $X^{n+1}$. Thus we have the analogue of Theorem 1.1 (a) for this case. As for part (b) of the theorem, Pogorelov claims in his preface to [13] that his formulas can be suitably modified for a suitable model of the constant negative curvature case. It seems entirely possible to us that the proof of rigidity in $\S 4$ could also be suitably modified to give the analogue of (b) for hyperbolic space case. However, we have not attempted this.

\subsection{Remarks}

(a) It may be mentioned that the fact that a compact manifold $M$ is immersed as a hypersurface of the hyperbolic space puts some restrictions on the curvature of $\boldsymbol{M}$. For instance, it has been shown by Amaral [2, p. 19] that under such hypotheses, some sectional curvature of $M$ is necessarily positive.

(b) Our methods do not apply for complete, non-compact hypersurfaces of a hyperbolic space to give a result similar to that of Sacksteder in [14]. Actually such a result will be of little topological interest in view of the following theorem $[1$, p. 453]: Given any open connected set $U$ in a sphere of dimension $n-1$, there exists a complete convex hypersurface $S$ in the hyperbolic space of dimension $n$ such that $S$ is homeomorphic with $U$.

\section{Bibliography}

[1] A. D. Alexandroff, Die innere Geometrie der konvexen Flächen, Moscow, 1948, in Russian. German translation, Akademie, Berlin, 1955.

[2] L. Amaral, Thesis at the University of California, Berkeley, 1964.

[ 3 ] L. Bianchi, Vorlesungen über Differentialgeometrie, German translation by $M$. Lukat, Leipzig, 1899.

[4] R. Bishop \& R. Crittenden, Geometry of manifolds, Academic Press, New York, 1964.

[ 5 ] S. S. Chern, Curves and surfaces in euclidean space, Studies in Global Geometry and Analysis, Math. Assoc. Amer., 1967, 16-56.

[6] S. S. Chern \& R. K. Lashof, On the total curvature of immersed manifolds. II, Mich. Math. J. 5 (1958) 5-12.

[7] M. P. do Carmo \& E. Lima, Isometric immersions with semi-definite second quadratic forms, to appear in Archiv der Mathematik.

[ 8 ] J. Hadamard, Sur certaines propriétés des trajectories en dynamique, J. Math. Pures Appl. 3 (1897) 331-387.

[9] J. Lelong-Ferrand, Géométrie différentielle, Masson, Paris, 1963.

[10] B. O'Neill, Isometric immersions which preserve curvature operators, Proc. Amer. Math. Soc. 13 (1962) 759-763. 
[11] A. V. Pogorelov, On the rigidity of general closed surfaces, Kiev, 1952, in Russian. German translation, Akademie, Berlin, 1957.

[12] —- Einige Untersuchungen zur Riemannschen Geometrie im Grossen, Kharkov University Press, 1957, in Russian. German translation, VEB Deutscher Verlag der Wissenschaften, Berlin, 1960.

[13] - Topics in the theory of surfaces in elliptic space, Kharkov University Press, 1960, in Russian. English translation, Gordon and Breach, New York, 1961.

[14] R. Sacksteder, On hypersurfaces with no negative sectional curvatures, Amer. J. Math. 82 (1960) 609-630.

[15] —, The rigidity of hypersurfaces, J. Math. Mech. 11 (1962) 929-940.

[16] J. van Heijenoort, On locally convex manifolds, Comm. Pure Appl. Math. 5 (1952) 845-852.

[17] K. Voss, Differentialgeometrie geschlossener Flächen in Euklidischen Raum. I, Jber. Deutsch. Math. Verein. 63 (1960) 117-136.

University of California, Berkeley

I.M.P.A., RIO DE JANEIRO, BRAZIL;

UNIVERSITY OF PENNSYlVANIA 